\title{
Factor structure and measurement invariance of the problematic mobile phone use questionnaire-short version across gender in Chinese adolescents and young adults
}

\author{
Ying-Ying Wang ${ }^{1,2,3}$, Jiang Long ${ }^{1,2,4}$, Yue-Heng Liu ${ }^{1,2}$, Tie-Qiao Liu ${ }^{1,2^{*}}$ (iD and Joël Billieux $x^{5,6^{*}}$
}

\begin{abstract}
Background: Problematic mobile phone use (PMPU) has become a public health issue in China, particularly in adolescents and young adults. The Problematic Mobile Phone Use Questionnaire-Short Version (PMPUQ-SV) is a validated instrument that measures multiple aspects of PMPU. The current study aimed to test the psychometric characteristics of a Chinese adaption of the PMPUQ-SV and examine its measurement invariance across gender.

Methods: A total of 2086 participants were recruited form nine schools (six undergraduate colleges and three vocational colleges) through an online platform. Measures included socio-demographic variables, patterns of mobile phone use, the Chinese version of the PMPUQ-SV (C-PMPUQ-SV), the Chinese version of the Smartphone Addiction Proneness Scale (C-SAPS), and the Depression Anxiety Stress Scales (DASS-21).

Results: Exploratory and confirmatory factor analyses conducted in two independent subsamples confirmed that the postulated dimensions fit the data well. Four items, judged as either outdated or not adapted to the Chinese context, performed poorly and were removed, resulting in a shorter 11-item scale. Convergent validity was established through correlations between emotional symptoms and the C-PMPUQ-SV and addictive smartphone use. Additional measurement invariance analyses showed that the scale performed largely similarly in male and female participants.

Conclusions: The present study demonstrated that the C-PMPUQ-SV is an adequate instrument to study various types of PMPU in Chinese adolescents and young adults. The updated 11-item scale shortens the evaluation time and is adapted to assess contemporary smartphone use.
\end{abstract}

Keywords: Mobile phone use, Problematic smartphone use, Mobile phone addiction, Smartphone addiction, Measurement invariance

\footnotetext{
*Correspondence: liutieqiao123@csu.edu.cn; Joel.Billieux@unil.ch

${ }^{1}$ Department of Psychiatry, The Second Xiangya Hospital, Central South

University, No.139, Renmin Middle Road, Changsha 410011, Hunan, China

${ }^{5}$ Institute of Psychology, University of Lausanne, Lausanne, Switzerland

Full list of author information is available at the end of the article
}

(C) The Author(s). 2020 Open Access This article is distributed under the terms of the Creative Commons Attribution 4.0 International License (http://creativecommons.org/licenses/by/4.0/), which permits unrestricted use, distribution, and reproduction in any medium, provided you give appropriate credit to the original author(s) and the source, provide a link to the Creative Commons license, and indicate if changes were made. The Creative Commons Public Domain Dedication waiver (http://creativecommons.org/publicdomain/zero/1.0/) applies to the data made available in this article, unless otherwise stated. 


\section{Background}

The development of information technology is unprecedented, and mobile phones have been updated to smartphones at a breathtaking pace in the past decade. In 2018, the number of smartphone subscribers worldwide exceeded 2.5 billion, and China accounted for $28.52 \%$ of the total (i.e., equivalent to the sum of users in all European countries combined) [1]. Because it allows people to utilize without the limitations of space and time, the smartphone penetrates the fields of health care [2-4] and education [5], as well as individuals' lives (e.g., communication, entertainment, and shopping) [6-8] with tremendous efficiency. Despite the convenience of mobile phones, however, the related adverse effects associated with problematic mobile phone use (PMPU) are noteworthy. PMPU has been broadly defined as the inability to regulate one's use of the mobile phone, which eventually involves negative consequences in daily life [9], including somatic discomfort [10, 11], sleep disturbance [12, 13], negative emotions and stress [14-19], poor academic performance [20, 21], low self-esteem [22, 23], and accidental injuries (e.g., motor vehicle accidents [24], bicycle crashes/near crashes [25], pedestrian collisions, and falls [26]).

Although the consensus is that PMPU has negative effects, an ongoing debate remains regarding its conceptualization and assessment. Various terms have been used to label this problematic behavior, including mobile phone dependence [27], mobile phone/smartphone addiction [28-30], and nomophobia [31]. Crucially, PMPU has often been conceptualized as a genuine addictive behavior, implying that most screening tools for this behavior (e.g., Smartphone Addiction Scale [32]) have been modeled after diagnostic criteria for substance use and gambling disorders [33, 34]. One recurrent criticism to such an approach is that some of these criteria are not necessarily valid indicators of pathological behaviors (i.e., they often fail to distinguish heavy but not problematic use from pathological use) [35]. Recycling these criteria to create screening tools is thus susceptible to overpathologization [33]. Beyond that, a potential limitation of most existing scales (e.g., Mobile Phone Problem Usage Scale [36]) is that they are unidimensional, despite the fact that other types of problematic use (e.g., antisocial use or risky use) have been identified $[9,37]$. In order to tackle the heterogeneous nature of PMPU, Billieux, Van der Linden, and Rochat [38] developed the Problematic Mobile Phone Use Questionnaire (PMPUQ), a 30-item scale that measures four distinct issues related to mobile phone use: (1) perceived dependence (PD), (2) prohibited (or antisocial) use (PU), (3) dangerous use (DU), and (4) financial problems. In recent years, the PMPUQ has been used in various studies to investigate the risk factors and correlates associated with PMPU [39-41].
Updated (e.g., DU not only concerns drivers, but also pedestrians or cyclists) and shorter versions of the scale have recently emerged to keep pace with the constant evolution of smartphones (e.g., the Financial Problems subscale has been removed, as it is now possible to make extensive use of smartphones with free apps) [18, 37, 42].

Calls have also emerged for an investigation into the problematic use of information and communication technologies to better understand its worldwide public health relevance from a cross-cultural perspective [43]. To accommodate this, Lopez-Fernandez and her colleagues [42] adapted a 15-item short version of the PMPUQ (PMPUQ-SV) and confirmed its factorial structure and measurement invariance across five different languages (i.e., French, German, Hungarian, Finnish, and Spanish). Yet to date, the PMPUQ-SV has not been adapted and tested in the context of East Asia, despite this region having the world's highest smartphone penetration rates. It is thus urgent to adapt such a scale to the East Asian context in order to initiate psychometrically sound cross-cultural research on PMPU related to Asian countries. Such research is also needed to determine whether the scale performs adequately in the East Asian context, which cannot be guaranteed: LopezFernandez et al.'s [42] study failed to validate some versions of the scale (i.e., Polish, Italian), suggesting that the postulated factor structure of the PMPUQ-SV may not necessarily be reproduced in all cultures. This could be due to important differences in the way that smartphones are used in different cultural contexts or countries [44].

Numerous studies have explored the consequences and correlates of PMPU in adolescents and young adults. This focus is explained as either being because these populations are "digital natives" (they grew up with these mobile technologies), or because adolescents and young adults are generally considered a vulnerable population for developmental reasons [45-47]. Yet to date, few studies have explored different problematic usage patterns (i.e., not merely related to addictive use) in Chinese youth. In such a context, the primary purpose of the present study was to develop a Chinese version of the PMPUQ-SV (C-PMPUQ-SV) proposed by LopezFernandez et al. [42]. To this end, a three-step procedure was undertaken. First, we applied exploratory factor analysis (EFA) and confirmatory factor analysis (CFA) to the C-PMPUQ-SV in two independent subsamples. Second, we tested the convergent validity against another scale that specifically assessed addictive use of the smartphone, while we explored the construct validity of the C-PMPUQ-SV through its relationships with emotional symptoms (stress, depression, anxiety), which were linked to PMPU in previous studies [48]. Third, because previous research emphasized gender differences in 
PMPU [49, 50], we also tested measurement invariance across gender. This last step is important, given the number of previous psychometric studies that validated the PMPU scale while overlooking gender-related measurement issues.

\section{Methods}

\section{Procedures and samples}

According to the school-running level, we first divided all higher education institutions of Changsha (which is one of the largest educational centers in Mainland China) into three categories (i.e., Key Universities, General Universities and Vocational Colleges), then we adopted the strategy of stratified random sampling, taking three schools from each level. Data were collected through an online platform (Qualtrics, Provo, UT) with the help of the teacher in charge of a class (class advisers). Researchers introduced the objectives of the study to the class advisers and trained them how to give instructions to the students. As the link was forwarded, a total of 4333 responders started the survey (i.e., clicked the link), but only those with a $100 \%$ completion rate and rated "excellent" by Qualtrics were retained for further analysis. In addition, 26 participants were excluded because their reported ages and educational level did not match those of the target sample (i.e., full-time undergraduate or below aged 14 to 25 years). The final sample size was 2086, including 409 (19.6\%) males and 1677 $(80.4 \%)$ females. The mean $( \pm S D)$ age for the total sample, males, and females was $18.30( \pm 1.30), 18.49( \pm 1.20)$, and $18.25( \pm 1.32)$, respectively.

\section{Ethics}

Given that some of the participants were younger than 18 years, we sent verbal informed consent to their parents (or legal guardians) with the help of the class advisers prior to the survey. We only obtained the verbal informed consent because the participants are almost residential students who meet their parents (or legal guardians) merely during the holidays. Anonymity and confidentiality were guaranteed during the whole progress of survey and data analysis, and no data regarding the identification of participants were collected, including their Internet Protocol address. The study protocol emphasized the foregoing irresistible clause (i.e., only verbal consent could be obtained) and was approved by the Ethics Committee of the Second Xiangya Hospital of Central South University. Some data, not related to the current study, were also collected and will be presented elsewhere.

\section{Instruments}

The Chinese version of the PMPUQ-SV (C-PMPUQ-SV).

The C-PMPUQ-SV was adapted from Lopez-Fernandez et al. [42] and comprises 15 items that assess three postulated factors: PD, DU, and PU. The items are scored on a 4-point Likert scale ranging from 1 (strongly agree) to 4 (strongly disagree) and some items have to be reversed before scoring. Higher scores reflect more serious PMPU. From standard scale-adapting procedures [51], one author (JL) first translated the French and English items into Chinese. Two authors (YHL and YYW), both with clinical research backgrounds and good proficiency in English, then back-translated the scale. One author, who is also the creator of the original version of the scale (JB), supervised the process and confirmed that the back-translated items corresponded to the original items. In accordance with recent work conducted to update the PMPUQ [36], some wording of items pertaining to the DU subscale (i.e., items $2,5,11,14$ ) was modified prior to the translation procedure to cover DU by both pedestrians and cyclists (e.g., looking at a smartphone while crossing a road). Indeed, research has shown that DU of mobile phones is no longer limited to drivers and that pedestrians are more and more putting themselves into risky situations while using smartphones [52, 53]. The three versions (i.e., French, English, and Chinese) of the 15-item PMPUQ-SV and descriptions of the items are provided in an additional file (see Additional file 1).

\section{The Chinese version of the smartphone addiction proneness scale (C-SAPS)}

The Smartphone Addiction Proneness Scale (SAPS) [30] is a self-reported instrument to assess symptoms of smartphone addiction (i.e., functional impairment, withdrawal, tolerance, online life orientation). It includes 15 items rated on a 4-point Likert scale $(1=$ strongly disagree, $2=$ disagree, $3=$ agree, $4=$ strongly agree), with higher scores implying higher smartphone addictive use. The Chinese version of the scale (C-SAPS) [54] was adapted prior to the current study by our team. The internal consistency of the total scale was 0.852 in the current sample, which corresponds to excellent reliability.

\section{Depression anxiety stress Scales-21 (DASS-21)}

The DASS-21 $[55,56]$ is one of the most used and reliable scales for measuring emotional symptoms worldwide. It has been translated into 50 languages [57], and the factor structure has been established in Chinese [58]. It measures three types of emotional symptoms: depression, anxiety, and stress. Each subscale contains seven items scored on a 4-point Likert scale, ranging from 0 (did not apply to me at all) to 3 (applied to me very much, or most of the time); higher scores indicate more emotional symptoms experienced in the past week. In the current sample, the internal consistency coefficient for the depression, anxiety, and stress subscale was $0.870,0.762$, and 0.834 , respectively, indicating good internal reliability. 


\section{Data analytic strategy}

Four consecutive statistical analysis steps were performed with SPSS 23.0 (IBM, 2014) and Mplus 7.4 (Muthén \& Muthén, 2015). First, an EFA was conducted on a randomly split half of the total sample (sample 1, $n=1043$ ). We used principal component analysis with Promax rotation to identify an optimal data-driven factor structure, as this method allows correlations between latent factors. Items that were found to be inconsistent with the original PMPUQ-SV, along with those that loaded equally on more than one factor, were removed.

Second, a series of CFAs were conducted on the other split half of the sample (sample 2, $n=1043$ ) to compare the fit of several competing models. Model 1 tested the original three-factor model containing 15 items [42]. Model 2 tested a two-factor model in which the PU subscale was not used because of the low internal consistency found in previous studies [18, 42]. Model 3 tested the 11-item model that was identified in our EFA. As the measures of the C-PMPUQ-SV items are not normally distributed (i.e., the skewness for each item ranged from 0.015 to 0.652 and the kurtosis ranged from 0.009 to 1.912 ), we used the Satorra-Bentler maximum likelihood mean adjusted estimator instead of the maximum likelihood estimator. To assess the fit of each model, we examined multiple fit indices [59], including chi-square, the root mean square error of approximation (RMSEA), the standardized root mean square residual (SRMR), the Tucker-Lewis index (TLI), and the comparative fit index (CFI). We simultaneously adopted CFI and TLI values of $\geq 0.90$ and an RMSEA value of $\leq 0.05$ as having a good fit [60]. Notably, as the chi-square is known to be highly influenced by the sample size [60], it was reported but not considered as a fit index in the present study.

Third, on the one hand, we examined reliability by analyzing the internal consistency of the adapted scale and its subscales. On the other hand, we calculated the Pearson's correlation coefficients between the CPMPUQ-SV, the C-SAPS, and the DASS- 21 to test convergent and construct validity. Both analyses were performed for the whole sample.

Last, we assessed measurement invariance across gender after the best structure was determined. We initially assessed the best-fit model in male and female groups separately, and then we tested configural invariance, metric invariance (or weak invariance), scalar invariance (or strong invariance), and error variance invariance (or strict invariance). More specifically (1), configural invariance tested whether the basic model structure of the latent variables was invariant across groups; (2) metric invariance, built on configural invariance, constrained factor loadings to be equivalent across groups; (3) scalar invariance, while assuming configural invariance and metric invariance to be established, tested whether the variable intercepts were equivalent across groups; and (4) error variance invariance, based on all of the previous types of invariance, set the error variance to be equal. We capitalized on fit index differences for RMSEA, SRMR, CFI, and TLI (i.e., $\triangle$ RMSEA, $\triangle$ SRMR, $\triangle \mathrm{CFI}$, and $\triangle \mathrm{TLI}$ ) as reference points, with a $P$-value of $<0.01$ indicating no difference, a $P$-value between 0.01 and 0.02 indicating moderate difference, and a $P$-value of $>0.02$ indicating an important difference [61, 62]. After having established measurement invariance, we computed a series of independent sample $t$-tests in order to examine gender differences regarding the various C-PMPUQ-SV subscales.

\section{Results}

\section{Exploratory factor analysis}

According to the values for the Kaiser-Meyer-Olkin measure of sampling adequacy and Bartlett's test of sphericity, the data were suitable for factorial analysis. Three consecutive rounds were necessary to remove the items that were inconsistent with the original structure or that loaded equally on more than one factor. In the first round, items 9 and 5, which did not load on the expected factor, were removed. In the second round, items 1 and 7 both loaded similarly on two factors (i.e., crossloadings were all above 0.5 ; see the values in parentheses in Table 1) and were removed. In the third round, we successfully identified a three-factor solution in which all factors had an eigenvalue above 1.0. The three factors accounted for $55.94 \%$ of the total variance, with DU accounting for $29.49 \%$, PD accounting for $14.87 \%$, and PU accounting for $11.58 \%$. The loading of each item on the corresponding factor ranged from 0.613 (item 12 belongs to the PU scale) to 0.836 (item 13 belongs to the PD scale). In this final round, no item was found to load similarly, or notably, on more than one factor. The entire EFA process is depicted in Table 1.

\section{Confirmatory factor analysis}

Table 2 illustrated the fit indices of three competing models tested with CFA in subsample 2 . The only one that can be considered as having a good fit is model 3 , which was derived from the EFA conducted in sample 1. Model 3 provided an optimum fit to the data (i.e., $\mathrm{S}-\mathrm{BX}^{2}=$ 134.716, $\mathrm{df}=41, P<0.01 ;$ RMSEA $=0.047 ; \quad$ CFI $=0.942$; $\mathrm{TLI}=0.922$; $\mathrm{SRMR}=0.41)$ compared with the other two models. For model 2, only the RMSEA and SRMR indices met the requirements, whereas model 1 presented with an overall poor fit. Model 3 was thus used for correlation and measurement invariance analyses.

\section{Reliability and validity}

As shown in Table 3, the internal consistency coefficients of the C-PMPUQ-CV and its three subscales were 
Table 1 Factor loadings of the C-PMPUQ-SV based on EFA in sample $1(n=1043)$

\begin{tabular}{|c|c|c|c|c|c|c|c|c|c|}
\hline \multirow[t]{2}{*}{ Item } & \multicolumn{3}{|c|}{ 1st round } & \multicolumn{3}{|c|}{ 2nd round } & \multicolumn{3}{|c|}{ 3rd round } \\
\hline & DU & PD & PU & $\overline{\mathrm{DU}}$ & PD & PU & $\overline{\mathrm{DU}}$ & $P D$ & PU \\
\hline $\mathrm{KMO}$ & \multicolumn{3}{|l|}{0.830} & \multicolumn{3}{|l|}{0.816} & \multicolumn{3}{|l|}{0.790} \\
\hline$x^{2}$ & \multicolumn{3}{|c|}{$3952.538 P<0.001$} & \multicolumn{3}{|c|}{$3053.816 P<0.001$} & \multicolumn{3}{|c|}{$2397.266 P<0.001$} \\
\hline 8 & \multicolumn{3}{|l|}{0.766} & \multicolumn{3}{|l|}{0.772} & \multicolumn{3}{|l|}{0.785} \\
\hline 11 & \multicolumn{3}{|l|}{0.749} & \multicolumn{3}{|l|}{0.744} & \multicolumn{3}{|l|}{0.737} \\
\hline 14 & \multicolumn{3}{|l|}{0.742} & \multicolumn{3}{|l|}{0.730} & \multicolumn{3}{|l|}{0.744} \\
\hline 2 & \multicolumn{3}{|l|}{0.731} & \multicolumn{3}{|l|}{0.760} & \multicolumn{3}{|l|}{0.780} \\
\hline 9 & \multicolumn{3}{|l|}{0.533} & $\times$ & & & \multicolumn{3}{|l|}{$x$} \\
\hline 13 & \multicolumn{3}{|c|}{0.733} & \multicolumn{3}{|c|}{0.789} & \multicolumn{3}{|c|}{0.836} \\
\hline 10 & \multicolumn{3}{|c|}{0.714} & \multicolumn{3}{|c|}{0.755} & \multicolumn{3}{|c|}{0.775} \\
\hline 7 & \multicolumn{3}{|c|}{0.649} & & 0.587 & $(0.541)$ & \multicolumn{3}{|c|}{$\times$} \\
\hline 1 & \multicolumn{3}{|c|}{0.609} & & 0.536 & $(0.576)$ & \multicolumn{3}{|c|}{$\times$} \\
\hline 4 & \multicolumn{3}{|c|}{0.586} & & 0.629 & & & 0.684 & \\
\hline 5 & & & 0.752 & & & $\times$ & & $x$ & \\
\hline 12 & & & 0.684 & & & 0.571 & & & 0.613 \\
\hline 3 & & & 0.646 & & & 0.675 & & & 0.714 \\
\hline 15 & & & 0.544 & & & 0.653 & & & 0.668 \\
\hline 6 & & & 0.536 & & & 0.632 & & & 0.637 \\
\hline
\end{tabular}

Note: C-PMPUQ-SV Chinese version of the Problematic Mobile Phone Use Questionnaire-Short Version, EFA exploratory factor analysis, DU dangerous use, $P D$ perceived dependence, $P U$ prohibited use, $K M O$ Kaiser-Meyer-Olkin measure of sampling adequacy, $X=$ Bartlett's test of sphericity approximate chi-square, values in parentheses $=$ the secondary factor loading, $x=$ items removed in the previous rotation

0.742 (total scale), 0.770 (DU subscale), 0.697 (PD subscale), and 0.574 (PU subscale). Regarding the convergent validity of the C-PMPUQ-SV, the correlation coefficients between the overall scale, its three subscales, and the total score of the C-SAPS in the whole sample $(n=2086)$ were $0.615(P<0.01$, total score), $0.691(P<0.01, \mathrm{PD}), 0.410$ $(P<0.01, \mathrm{DU})$, and $0.251(P<0.01, \mathrm{PU})$. Other than the PU subscale and anxiety, the C-PMPUQ-SV and its subscales were significantly positively correlated with the three types of emotional symptoms (all $P_{\mathrm{s}}<0.01$ ).

\section{Measurement invariance across gender}

Table 4 depicted the entire verification process for measurement invariance. First, as the baseline, model A

Table 2 Comparison of goodness-of-fit indices and models in sample $2(n=1043)$

\begin{tabular}{llllllll}
\hline Model & S-B $X^{2}$ & df & $P$ & CFI & TLI & RMSEA (90\% Cl) & SRMR \\
\hline
\end{tabular}

\begin{tabular}{lllllllll}
\hline Model 1 & 746.678 & 87 & $<0.01$ & 0.752 & 0.701 & 0.085 & $(0.080-0.091)$ & 0.087
\end{tabular}

Model $2 \quad 234.397 \quad 34<0.01 \quad 0.885 \quad 0.848 \quad 0.075(0.066-0.084) \quad 0.060$

$\begin{array}{lllllllll}\text { Model } 3 & 144.638 & 41 & <0.01 & 0.942 & 0.922 & 0.047 & (0.038-0.056) & 0.041\end{array}$

Note: $S-B X^{2}$ Satorra-Bentler corrected chi-square, $d f$ degrees of freedom, CFI comparative fit index, TLI Tucker-Lewis index, RMSEA root mean square error of approximation, Cl confidence interval, SRMR standardized root mean square residual which was derived from our EFA, without any parameter constrictions being successfully set, established configural invariance $(\mathrm{CFI}=0.936$, TLI $=0.914$, RMSEA $[90 \%$ confidence interval $]=0.050 \quad[0.044,0.056], \quad S R M R=$ 0.043), indicating that the pattern of the latent variables was consistent across gender. As stated in the Data Analytic Strategy section, we tested the multiple fit indices for both gender groups, further supporting that the baseline model had acceptable fit for both males and females (see last two rows in Table 4).

Second, model B was set on the basis of model A, which constricted the equality of factor loadings between the two gender groups. Results $(\Delta \mathrm{CFI}=0.000, \Delta \mathrm{TLI}=0.007$, $\triangle$ RMSEA $=0.002$, and $\triangle$ SRMR $=0.001)$ support that the factor loadings of each latent variable between different groups were comparable, thus implying that the metric invariance (or weak invariance) was established.

Further, on the basis of model B, model $\mathrm{C}$ hypothesized that the item intercepts are equal across gender. The results showed that the model did not deteriorate significantly $(\triangle \mathrm{CFI}=0.007, \quad \Delta \mathrm{TLI}=0.001, \quad \triangle \mathrm{RMSEA}=$ 0.000 , and $\triangle S R M R=0.002$ ), indicating that scale invariance (or strong invariance) was supported.

Finally, after scalar invariance, we tested whether the error variance had cross-group equivalence. All of the $\Delta$ values except for $\triangle$ CFI $(0.015)$ were well below 0.01 $(\Delta \mathrm{TLI}=0.007, \Delta \mathrm{RMSEA}=0.002$, and $\triangle \mathrm{SRMR}=0.003)$. Taken together, the results partially supported error variance invariance (or strict invariance).

It is worth stressing that in order to control the influence of age on our results, we divided the subjects into two groups according their age (minors and adults) and tested measurement invariance across gender in each group. Results showed that age had no significant influence on the results (see Additional file 2).

\section{Gender differences of the PMPU}

Given that measurement invariance across gender was established, we tested gender differences in the CPMPUQ-SV and its subscales. Using the whole sample $(n=2086)$, we found that females scored significantly higher than males on the PD subscale $(P<0.05)$, but there was no significant difference between the overall scale and the other two subscales. The results were depicted in Table 5.

\section{Discussion}

This study is the first to examine the psychometric characteristics of the PMPUQ-SV in the East Asian context. Capitalizing on EFA and CFA conducted in two independent subsamples, the current study reproduced the three-factor structure shown in previous studies [18, 42]. However, the updated scale was reduced to 11 items following the exclusion of 4 items that performed poorly in 
Table 3 Internal consistency, reliability, and convergent and construct validity

\begin{tabular}{|c|c|c|c|c|c|c|c|c|}
\hline Measure & C-PMPUQ-SV & DU & PD & $\mathrm{PU}$ & C-SAPS & Stress & Depression & Anxiety \\
\hline C-PMPUQ-SV & $(0.742)$ & & & & & & & \\
\hline DU & $0.794^{* *}$ & $(0.770)$ & & & & & & \\
\hline PD & $0.725^{* *}$ & $0.417^{* *}$ & $(0.697)$ & & & & & \\
\hline PU & $0.613^{* *}$ & $0.159^{* *}$ & $0.219^{* *}$ & $(0.574)$ & & & & \\
\hline C-SAPS & $0.615^{* *}$ & $0.410^{* *}$ & $0.691^{* *}$ & $0.251^{* *}$ & $(0.852)$ & & & \\
\hline Stress & $0.319^{* *}$ & $0.272^{* *}$ & $0.297^{* *}$ & $0.105^{* *}$ & $0.352^{* *}$ & $(0.834)$ & & \\
\hline Depression & $0.311^{* *}$ & $0.240^{* *}$ & $0.274^{* *}$ & $0.149^{* *}$ & $0.297^{* *}$ & $0.751^{* *}$ & $(0.870)$ & \\
\hline Anxiety & $0.211^{* *}$ & $0.193^{* *}$ & $0.218^{* *}$ & 0.030 & $0.255^{* *}$ & $0.718^{* *}$ & $0.647^{* *}$ & $(0.762)$ \\
\hline
\end{tabular}

Note: ** $P<0.01, C-P M P U Q-S V$ Chinese version of the Problematic Mobile Phone Use Questionnaire-Short Version; $D U$ dangerous use; $P D$ perceived dependence; $P U$ prohibited use; C-SAPS Chinese version of the Smartphone Addiction Proneness Scale

the current population. Internal consistency of the various subscales was globally acceptable, although the PU subscale presented with a low Cronbach's $\alpha$ value. Statistically significant correlations were found between the C-PMPUQ-SV and the C-SAPS and between the CPMPUQ-SV and the DASS-21, which supported convergent and construct validity. Moreover, this study is the first to conduct measurement invariance assessment of the PMPUQ-SV across gender by using multigroup CFA. Results provided evidence that there was measurement invariance across gender, and the latent means comparison demonstrated that females had significantly higher PD scores than males did.

EFA determined a three-factor solution, which was consistent with the structure of the original scale, supporting that PMPU is indeed a multidimensional construct $[9,38]$. However, this updated scale differs from the original one in the sense that some items were found to be less adapted to the current context. Item 5 ("I try to avoid using my mobile phone when driving on the motorway") refers to the dangerous behavior of the responders in driving on the motorway, which is not in line with the situation in China, as Chinese students have less driving experience before graduation. Item 9 ("I use my mobile phone where it is forbidden to do so") fell into the DU subscale instead of the expected PU subscale. Both item 1 and item 7 had very high crossloadings, indicating that these two items might have low discriminative power [42]. Eventually, we retained 11 items categorized under three factors, their cumulative contribution explaining $55.94 \%$ of the variance. To validate the C-PMPUQ-SV derived from the EFA, we conducted a CFA in the second split half of the sample and compared various competitive models (i.e., the original questionnaire and the adapted one that excluded the PU subscale) suggested in previous research [36]. The model inspired from the EFA fit the data best. It is worth noting that the factorial structure found in the current study did not entirely match that of the original scale, a problem already emphasized for other adaptations of the scale in another cross-cultural study [42]. This further supports that PMPU may have items that are specifically applicable to different regions or countries [18, 42], such as China in the current study. This also supports the necessity of updating scales developed to assess mobile phone use at times when smartphones were not yet available (the original PMPUQ was developed in 2006 and published in 2008) [37].

In terms of internal consistency, the total scale and the two subscales reach acceptable values, whereas the PU subscale (Cronbach's $\alpha=0.574$ ) is characterized by relatively poor internal consistency. This finding is similar to what was previously found for the English version (Cronbach's $\alpha=0.56$ [18]) and Finnish version (Cronbach's $\alpha=0.59$, [18]) of the PMPUQ-SV. This lower reliability could be related to differences prohibition among

Table 4 Multiple fit indices for measurement invariance across gender

\begin{tabular}{|c|c|c|c|c|c|c|c|c|c|c|c|c|c|}
\hline Model & $S-B x^{2}$ & $d f$ & CFI & TLI & RMSEA (90\% Cl) & SRMR & $\Delta x^{2}$ & $\Delta d f$ & $P$ & $\Delta \mathrm{CFI}$ & $\Delta \mathrm{TLI}$ & $\triangle \mathrm{RMSEA}$ & $\triangle \mathrm{SRMR}$ \\
\hline Model A & 294.888 & 82 & 0.936 & 0.914 & $0.050(0.044-0.056)$ & 0.043 & - & - & $<0.01$ & - & - & - & - \\
\hline Model B & 303.730 & 90 & 0.936 & 0.921 & $0.048(0.042-0.054)$ & 0.044 & 5.960 & 8 & 0.750 & 0.000 & 0.007 & 0.002 & 0.001 \\
\hline Model C & 334.419 & 98 & 0.929 & 0.920 & $0.048(0.042-0.054)$ & 0.046 & 32.002 & 8 & $<0.001$ & 0.007 & 0.001 & 0.000 & 0.002 \\
\hline Model D & 395.797 & 109 & 0.914 & 0.913 & $0.050(0.045-0.056)$ & 0.049 & 59.557 & 11 & $<0.001$ & 0.015 & 0.007 & 0.002 & 0.003 \\
\hline Model $_{\text {M }}$ & 99.236 & 41 & 0.928 & 0.903 & $0.059(0.044-0.074)$ & 0.058 & - & - & $<0.01$ & - & - & - & - \\
\hline Model $_{F}$ & 201.978 & 41 & 0.937 & 0.916 & $0.048(0.042-0.055)$ & 0.039 & - & - & $<0.01$ & - & - & - & - \\
\hline
\end{tabular}

Note: S-BX2 Satorra-Bentler corrected chi-square; $d f$ degrees of freedom; CFI comparative fit index; TLI Tucker-Lewis index; RMSEA root mean square error of approximation; $C l$ confidence interval; SRMR standardized root mean square residual; $\Delta$ deviation magnitude of fit indices 
Table 5 Scores on the total C-PMPUQ-SV and three subscales separated by gender

\begin{tabular}{lllll}
\hline Scale & Male $(\mathrm{M} \pm \mathrm{SD})$ & Female $(\mathrm{M} \pm \mathrm{SD})$ & $t$ & $P$ \\
\hline C-PMPUQ-SV & $23.78 \pm 4.52$ & $23.87 \pm 3.93$ & 0.333 & 0.739 \\
PD & $6.46 \pm 1.73$ & $6.66 \pm 1.57$ & 2.094 & 0.037 \\
DU & $8.74 \pm 2.61$ & $8.61 \pm 2.18$ & -0.908 & 0.365 \\
PU & $8.59 \pm 2.20$ & $8.60 \pm 1.65$ & 0.097 & 0.923 \\
\hline
\end{tabular}

Note: C-PMPUQ-SV Chinese version of the Problematic Mobile Phone Use Questionnaire-Short Version; $P D$ perceived dependence; $D U$ dangerous use; $P U$ prohibited use

various countries and jurisdictions (e.g., using the mobile phone in a library or on public transportation may or may not be acceptable, depending on national regulations). Another potential explanation is related to the low number of items that constitute this subscale, as it is known that Cronbach's $\alpha$ is highly sensitive to the number of items in the scale $[63,64]$.

Correlation analysis was conducted to investigate the convergent validity of the scale. First, it appeared that the various facets of the C-PMPUQ-SV significantly correlated with the C-SAPS, the latter questionnaire being designed to assess and diagnose the addictive use of smartphones [30]. Unsurprisingly, the C-PMPUQ-SV subscale that presented the highest correlation $(r=0.69)$ with the C-SAPS was the PD subscale. Indeed, the PD subscale includes items related to loss of control or preoccupation toward mobile phone use, implying that a strong link with a measure of addictive use of the smartphone was expected and necessary to ascertain convergent validity of the C-PMPUQ-SV. Second, we also considered the relationships with emotional symptoms as assessed by the DASS-21 (i.e., depression, anxiety, and stress) to support the construct validity of the scale, as PMPU has been linked to emotional disorders in much previous research [48]. Most correlations between the CPMPUQ-SV and the DASS-21 were significant. Such results were consistent with past findings showing that emotional symptoms positively correlated with PMPU $[16,17,37,44,47,65]$. Overall, these results support the construct validity of the C-PMPUQ-SV.

A last objective of the current study was to examine measurement invariance across gender. The analyses conducted showed that the C-PMPUQ-SV was fully invariant regarding configural, metric, and scalar analyses (i.e., the changes in all fit indices were well below the prespecified cutoff $\Delta$ values), although strict invariance was only partially supported (i.e., all differences except $\Delta C F I$ were within the ideal range). The measurement invariance analyses suggested that the scale performs similarly well for males and females. We thus decided to compare the various C-PMPUQ-SV scales in terms of gender. A series of independent sample $t$-tests showed that gender differences are limited to the PD subscale, where females scored significantly higher than males. This finding was congruent with previous evidence [38, 44] and supported the idea that females are more prone to use the mobile phone to regulate negative mood states $[38,66,67]$. In contrast to previous studies that showed more frequent DU in men [38], no gender difference occurred regarding DU in the present study. Our results are hardly comparable, however, with those of Billieux et al. [38] because DU in the current study was not only related to reckless driving, but also to risky cycling or pedestrian behaviors.

Several limitations of the study have to be acknowledged. First, the participants were selected through a convenience sampling method, which did not guarantee representativeness and led to gender imbalance. Fortunately, the relatively large sample recruited counterbalanced this limitation and ensured adequacy regarding the study objectives and the statistical analyses performed. Second, this study was conducted by using a retrospective self-report. The participants may thus have overestimated or underestimated their actual and problematic use of the mobile phone. Further research capitalizing on both self-report and mobile phone tracking should be undertaken, as the two types of measurement are not necessarily correlated [68].

\section{Conclusions}

Despite these limitations, the current study showed that the C-PMPUQ-SV is a promising instrument to study various types of PMPU in the Chinese context, a country where hazardous mobile phone use is considered a public health issue and much research on its causes, consequences, and prevention is conducted. Our findings could provide guidance for the healthy development of adolescents. On the one hand, it opens up a new avenue for measuring the multiple style of PMPU in college students, which is helpful for educational institutions to understand the current situation of PMPU of the students and propose effective interventions to reduce dependence on mobile phones, especially for girls. On the other hand, it can be targeted to guide college students to use mobile phones reasonably, such as reduce the dangerous use behaviors (e.g., staring at the screen while crossing), so as to reduce the potential traffic accidents or personal injuries.

\section{Supplementary information}

Supplementary information accompanies this paper at https://doi.org/10. 1186/s12888-020-2449-0.

Additional file 1. Provides the French version (Table S1), English version (Table S2), and Chinese version (Table S3) of the 15-item PMPUQ-SV and describes item scoring

Additional file 2. Measurement invariance across gender aged 18 and below (Table S4) and Measurement invariance across gender aged above 18 (Table S5). 


\section{Abbreviations}

CFA: Confirmatory factor analysis; CFI: Comparative fit index; C-PMPUQSV: Chinese version of the Problematic Mobile Phone Use QuestionnaireShort Version; C-SAPS: Chinese version of the Smartphone Addiction Proneness Scale; DASS-21: Depression Anxiety Stress Scales-21; DU: Dangerous use; EFA: Exploratory factor analysis; PD: Perceived dependence; PMPU: Problematic mobile phone use; PMPUQ: Problematic Mobile Phone Use Questionnaire; PMPUQ-SV: Problematic Mobile Phone Use Questionnaire-Short Version; PU: Prohibited use; RMSEA: root mean square error of approximation; SAPS: Smartphone Addiction Proneness Scale; SRMR: Standardized root mean square residual; TLI: Tucker-Lewis index

\section{Acknowledgments}

We sincerely thank all of the participants and class advisers for their support and cooperation. We also thanks to Barbara Every, ELS, of BioMedical Editor, for English language editing of this article.

\section{Authors' contributions}

$L T Q, L J$, and JB designed the study. WYY and $L J$ elaborated the online survey and collected the data. $L J$ and LYH managed the translation process of the PMPUQ-SV in Chinese under the supervision of $\mathrm{JB}$, who is also the creator of the original version scale. WYY conducted the statistical analyses and interpreted the results. WYY and JB wrote the initial draft of the article. $L T Q, L$ and $L Y H$ reviewed and edited the manuscript. All authors approved the final version of the manuscript.

\section{Funding}

The current study was supported by grants from the National Key R\&D Program of China (2017YFC1310400), the National Natural Science Foundation of China (81371465 and 81671324) and the Research Foundation of Education Bureau of Hunan Province (17C0122). The sponsors had no role in the study design, survey process, data analysis, and manuscript preparation.

\section{Availability of data and materials}

The data sets used and/or analyzed during the current study are available from the corresponding author on reasonable request.

\section{Ethics approval and consent to participate}

Given that some of the participants aged under 18, we sent verbal informed consent to their parents (or legal guardians) with the help of the class advisers prior to the survey. We only obtained the verbal consent through message because the participants are almost residential students who meet their parents (or legal guardians) merely during the holidays. The study protocol emphasized the foregoing irresistible clause (i.e., only verbal consent could be obtained) and was approved by the Ethics Committee of the Second Xiangya Hospital of Central South University.

\section{Consent for publication}

Not applicable.

\section{Competing interests}

The authors declare that they have no competing interests.

\section{Author details}

'Department of Psychiatry, The Second Xiangya Hospital, Central South University, No.139, Renmin Middle Road, Changsha 410011, Hunan, China ${ }^{2}$ The China National Clinical Research Center on Mental Health Disorders (Xiangya), Chinese National Technology Institute on Mental Disorders, Hunan Key Laboratory of Psychiatry and Mental Health, Mental Health Institute of Central South University, No.139, Renmin Middle Road, Changsha 410011, Hunan, China. ${ }^{3}$ Changsha Normal University, Changsha, Hunan, People's Republic of China. ${ }^{4}$ Laboratory for Experimental Psychopathology, Psychological Science Research Institute, Université Catholique de Louvain, Louvain-la-Neuve, Belgium. ${ }^{5}$ Institute of Psychology, University of Lausanne, Lausanne, Switzerland. ${ }^{6}$ Addictive and Compulsive Behaviors Lab (ACB - Lab), Institute for Health and Behavior, University of Luxembourg, Esch-sur-Alzette, Luxembourg.
Received: 30 September 2019 Accepted: 21 January 2020

Published online: 30 January 2020

\section{References}

1. Statista. Number of smartphone users in China from 2017 to 2023 (in millions). 2019. https://www.statista.com/statistics/467160/forecast-ofsmartphone-users-in-china/. Accessed 3 Sept 2019.

2. Fortuna KL, Lohman MC, Gill LE, Bruce ML, Bartels SJ. Adapting a psychosocial intervention for smartphone delivery to middle-aged and older adults with serious mental illness. Am J Geriatric Psychiat. 2017;25(8): 819-28.

3. Hidalgo-Mazzei D, Reinares M, Mateu A, Juruena MF, Young AH, Pérez-Sola $V$, et al. Is a SIMPLe smartphone application capable of improving biological rhythms in bipolar disorder? J Affect Disord. 2017;223:10-6.

4. Achtyes ED, Ben-Zeev D, Luo ZH, Mayle H, Burke B, Rotondi AJ, et al. Offhours use of a smartphone intervention to extend support for individuals with schizophrenia spectrum disorders recently discharged from a psychiatric hospital. Schizophrenia Res. 2019;206:200-8.

5. O'Connor S, Andrews T. Smartphones and mobile applications (apps) in clinical nursing education: a student perspective. Nurse Educ Today. 2018; 69:172-8.

6. Holmes A, Byrne A, Rowley J. Mobile shopping behaviour: insights into attitudes, shopping process involvement and location. Int J Retail Distrib Manag. 2013:42(1):25-39.

7. Fuentes C, Svingstedt A. Mobile phones and the practice of shopping: a study of how young adults use smartphones to shop. J Retail Consum Serv. 2017;38:137-46

8. Hans G. Towards a sociological of the mobile phone. In: Sociology in Switzerland: sociology of the mobile phone. Online Publications. 2004 http://socio.ch/mobile/t_geser1.pdf. Accessed 3 Sept 2019.

9. Billieux J. Problematic use of the mobile phone: a literature review and a pathways model. Curr Psychiatr Rev. 2012;8(4):299-307.

10. Ali M, Asim M, Danish SH, Ahmad F, lqbal A, Hasan SD. Frequency of De Quervain's tenosynovitis and its association with SMS texting. Muscles Ligaments Tendons J. 2014;4(1):74-8.

11. Aggarwal K. Twenty-six percent doctors suffer from severe mobile phoneinduced anxiety: excessive use of mobile phone can be injurious to your health. Indian J Clin Pract. 2013;24(1):7-9.

12. Thomée S, Härenstam A, Hagberg M. Mobile phone use and stress, sleep disturbances, and symptoms of depression among young adults-a prospective cohort study. BMC Public Health. 2011;11:66.

13. Sahin S, Ozdemir K, Unsal A, Temiz N. Evaluation of mobile phone addiction level and sleep quality in university students. Pak J Med Sci. 2013;29(4):913-8

14. Leung L. Linking psychological attributes to addiction and improper use of the mobile phone among adolescents in Hong Kong. J Child Media. 2008; 2(2):93-113

15. Yen CF, Tang TC, Yen JY, Lin HC, Hung CF, Liu SC, et al. Symptoms of problematic cellular phone use, functional impairment and its association with depression among adolescents in southern Taiwan. J Adolesc. 2009;32: $863-73$

16. Chiu SI. The relationship between life stress and smartphone addiction on Taiwanese university student: a mediation model of learning self-efficacy and social self-efficacy. Comput Human Behav. 2014;34:49-57.

17. Demirci $K$, Akgönül M, Akpinar A. Relationship of smartphone use severity with sleep quality, depression, and anxiety in university students. J Behav Addict. 2015;4(2):85-92.

18. Lopez-Fernandez O, Männikkö N, Kääriäinen M, Griffiths MD, Kuss DJ. Mobile gaming and problematic smartphone use: a comparative study between Belgium and Finland. J Behav Addict. 2018;7(1):88-99.

19. Wilcockson TDW, Osborne AM, Ellis DA. Digital detox: the effect of smartphone withdrawal on mood, anxiety, and craving. Addict Behav. 2019; 99:106013.

20. Dos B. The relationship between mobile phone use, metacognitive awareness and academic achievement. Eur Educ Res J. 2014;3(4):192-200.

21. Lepp A, Barkley JE, Karpinski AC. The relationship between cell phone use, academic performance, anxiety, and satisfaction with life in college students. Comput Human Behav. 2013;31:343-50.

22. $\mathrm{Ha} J \mathrm{H}, \mathrm{Chin} \mathrm{B}$, Park DH, Ryu SH, YuJ. Characteristics of excessive cellular phone use in Korean adolescents. CyberPsychol Behav. 2008;11(6):783-4 
23. Yang YS, Yen JY, Ko CH, Cheng CP, Yen CF. The association between problematic cellular phone use and risky behaviors and low self-esteem among Taiwanese adolescents. BMC Public Health. 2010;10:217.

24. Pearson MR, Murphy EM, Doane AN. Impulsivity-like traits and risky driving behaviors among college students. Accid Anal Prev. 2013;53:142-8.

25. Ichikawa M, Nakahara S. Japanese high school students' usage of mobile phones while cycling. Traffic Inj Prev. 2014;9(1):42-7.

26. Tao SM, Wu XY, Wan YH, Zhang SC, Hao JH, Tao FB. Interactions of problematic mobile phone use and psychopathological symptoms with unintentional injuries: a school-based sample of Chinese adolescents. BMC Public Health. 2016;16:88

27. Toda M, Monden K, Kubo K, Morimoto K. Mobile phone dependence and health-related lifestyle of university students. Soc Behav Pers. 2006;34(10): 1277-84.

28. Chóliz M. Mobile phone addiction: a point of issue. Addiction. 2010;105: 373-5.

29. Kuss D. Mobile phone addiction: evidence from empirical research. Eur Psychiatry. 2017;45(Suppl):26-7.

30. Kim D, Lee YH, Lee JY, Nam JEK, Chung YJ. Development of Korean smartphone addiction proneness scale for youth. PLoS One. 2014;9(5): e97920.

31. King ALS, Valença AM, Silva ACO, Baczynski T, Carvalho MR, Nardi AE. Nomophobia: dependency on virtual environments or social phobia? Comput Human Behav. 2013;29(1):140-4.

32. Kwon M, Lee JY, Won WY, Park JW, Min JA, Hahn C, et al. Development and validation of a smartphone addiction scale (SAS). PLoS One. 2013;8(2): e56936.

33. Billieux J, Schimmenti A, Khazaal Y, Maurage P, Heeren A. Are we overpathologizing everyday life? A tenable blueprint for behavioral addiction research. J Behav Addict. 2015;4:119-23.

34. Starcevic $V$. Tolerance and withdrawal symptoms may not be helpful to enhance understanding of behavioural addictions: letter to the editor. Addiction. 2016;111(7):1307-8.

35. Billieux J, Flayelle M, Rumpf HJ, Stein DJ. High involvement versus pathological involvement in video games: a crucial distinction for ensuring the validity and utility of gaming disorder. Curr Addict Rep. 2019;6(3):323-30.

36. Bianchi A, Phillips JG. Psychological predictors of problem mobile phone use. CyberPsychol Behav. 2005;8:39-51.

37. Kuss DJ, Harkin L, Kanjo E, Billieux J. Problematic smartphone use: investigating contemporary experiences using a convergent design. Int J Environ Res Public Health. 2018;15(1):142.

38. Billieux J, Van Der Linden M, Rochat L. The role of impulsivity in actual and problematic use of the mobile phone. Appl Cogn Psychol. 2008;22:1195-210.

39. Billieux J, Gay P, Rochat L, Van Der Linden M. The role of urgency and its underlying psychological mechanisms in problematic behaviours. Behav Res Ther. 2010;48:1085-96.

40. Shrivastava A, Sharma MK, Marimuthu P. Internet addiction at workplace and it implication for workers life style: exploration from southern India. Asian J Psychiatry. 2018;32:151-5.

41. Kuss DJ, Kanjo E, Crook-Rumsey M, Kibowski F, Wang GY, Sumich A. Problematic mobile phone use and addiction across generations: the roles of psychopathological symptoms and smartphone use. J Technol Behav Sci. 2018;3(3):141-9.

42. Lopez-Fernandez O, Kuss DJ, Pontes HM, Griffiths MD, Dawes C, Justice LV, et al. Measurement invariance of the short version of the Problematic Mobile Phone Use Questionnaire (PMPUQ-SV) across eight languages. Int J Environ Res Public Health. 2018;15(6):1213.

43. World Health Organization. Public health implications of excessive use of the internet, computers, smartphones and similar electronic devices: meeting report. Foundation for Promotion of Cancer Research, National Cancer Research Center, Tokyo, Japan, 27-29 Aug 2014. Geneva, Switzerland: World Health Organization; 2015

44. Lopez-Fernandez O, Kuss DJ, Romo L, Morvan Y, Kern L, Graziani P, et al. Self-reported dependence on mobile phones in young adults: a European cross-cultural empirical survey. J Behav Addict. 2017:6(2):168-77.

45. Lopez-Fernandez O, Honrubia-Serrano L, Freixa-Blanxart M, Gibson W. Prevalence of problematic mobile phone use in British adolescents. Cyberpsychol Behav Soc Netw. 2014;17(2):91-8.

46. Livingstone S, Smith PK. Annual research review: harms experienced by child users of online and mobile technologies: the nature, prevalence and management of sexual and aggressive risks in the digital age. J Child Psychol Psychiatry. 2014;55(6):635-54

47. Long J, Liu TQ, Liao YH, Qi C, He HY, Chen SB, et al. Prevalence and correlates of problematic smartphone use in a large random sample of Chinese undergraduates. BMC Psychiatry. 2016;16(1):408.

48. Elhai JD, Dvorak RD, Levine JC, Hall BJ. Problematic smartphone use: a conceptual overview and systematic review of relations with anxiety and depression psychopathology. J Affect Disord. 2017;207:251-9.

49. Augner C, Hacker GW. Associations between problematic mobile phone use and psychological parameters in young adults. Int J Public Health. 2012;57: 437-41.

50. Jiang ZC. Zhao, XX. Self-control and problematic mobile phone use in Chinese college students: the mediating role of mobile phone use patterns BMC Psychiatry. 2016;16:416.

51. Brislin RW. Back-translation for cross-cultural research. J Cross-Cult Psychol. 1970;1(3):185-216.

52. Nasar J, Hecht P, Wener R. Mobile telephones, distracted attention, and pedestrian safety. Accid Anal Prev. 2008;40:69-75.

53. Stavrinos D, Byington KW, Schwebel DC. Distracted walking: cell phones increase injury risk for college pedestrians. J Saf Res. 2011;42(2):101-7.

54. Wang YY, Liu YH, Long J, Liu TQ. Psychometric characteristics of SAPS among Chinese students: a cross-sectional study. Unpublished technical report. Changsha, China: Central South University; 2019.

55. Lovibond PF, Lovibond SH. The structure of negative emotional states: comparison of the depression anxiety stress scales (DASS) with the Beck depression and anxiety inventories. Behav Res Ther. 1995:33(3):335-43.

56. Lovibond SH, Lovibond PF. Manual for the depression anxiety stress scales. 2nd ed. Psychology Foundation of Australia: Sydney, NSW; 1995.

57. Lovibond PF. DASS translations. Psychology Foundation of Australia 2018. http://www2.psy.unsw.edu.au/dass//translations.htm. Accessed 4 Sept 2019.

58. Moussa MT, Lovibond PF, Laube R. Psychometric properties of a Chinese version of the 21-item depression anxiety stress scales (DASS21). Sydney, NSW: Transcultural Mental Health Centre. Cumberland Hospital; 2001

59. Kline RB. Principles and practice of structural equation modeling. 3rd ed. New York, NY: The Guilford Press; 2011.

60. Rigon EE. A necessary and sufficient identification rule for structural models estimated in practice. Multivariate Behav Res. 1995:30(3):359-83.

61. Cheung GW, Rensvold RB. Evaluating goodness-of-fit indexes for testing measurement invariance. Struct Equ Modeling. 2002:9:233-55.

62. Meade AW, Johnson EC, Braddy PW. Power and sensitivity of alternative fit indices in tests of measurement invariance. J Appl Psychol. 2008:93:568-92.

63. Cortina JM. What is coefficient alpha? An examination of theory and applications. J Appl Psychol. 1993;78(1):98-104.

64. Helmstadter GC. Principles of psychological measurement. New York: Appleton-Century-Crofts; 1964

65. Wang JL, Wang HZ, Gaskin J, Wang LH. The role of stress and motivation in problematic smartphone use among college students. Comput Human Behav. 2015:53:181-8.

66. Geser H. Are girls (even) more addicted? Some gender patterns of cell phone usage. In: Sociology in Switzerland: sociology of the mobile phone 2006. http://socio.ch/mobile/t_geser3.htm. Accessed 4 Sept 2019.

67. Sánchez-Martínez M, Otero A. Factors associated with cell phone use in adolescents in the community of Madrid (Spain). CyberPsychol Behav. 2009; 12(2):131-7.

68. Lee $H$, Ahn H, Nguyen TG, Choi SW, Kim DJ. Comparing the self-report and measured smartphone usage of college students: a pilot study. Psychiatry Investig. 2017:14(2):198-204.

\section{Publisher's Note}

Springer Nature remains neutral with regard to jurisdictional claims in published maps and institutional affiliations. 